\title{
Foot Or Leg Hypoesthesia
}

National Cancer Institute

\section{Source}

National Cancer Institute. Foot Or Leg Hypoesthesia. NCI Thesaurus. Code C50571.

Consisting of abnormally decreased sensitivity, particularly to touch in the foot or leg. 